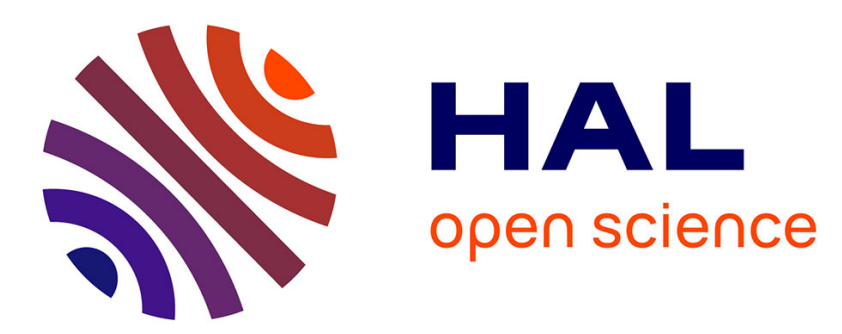

\title{
High fidelity force feedback facilitates manual injection in biological samples
}

Abdenbi Mohand Ousaid, Sinan Haliyo, Stephane Regnier, Vincent Hayward

\section{To cite this version:}

Abdenbi Mohand Ousaid, Sinan Haliyo, Stephane Regnier, Vincent Hayward. High fidelity force feedback facilitates manual injection in biological samples. IEEE Robotics and Automation Letters, 2020, 5 (2), pp.1758-1763. 10.1109/LRA.2020.2969940 . hal-03190990

\section{HAL Id: hal-03190990 \\ https://hal.science/hal-03190990}

Submitted on 26 Apr 2021

HAL is a multi-disciplinary open access archive for the deposit and dissemination of scientific research documents, whether they are published or not. The documents may come from teaching and research institutions in France or abroad, or from public or private research centers.
L'archive ouverte pluridisciplinaire HAL, est destinée au dépôt et à la diffusion de documents scientifiques de niveau recherche, publiés ou non, émanant des établissements d'enseignement et de recherche français ou étrangers, des laboratoires publics ou privés.

\section{(c)(1)}

Distributed under a Creative Commons Attribution| 4.0 International License 


\title{
High Fidelity Force Feedback Facilitates Manual Injection in Biological Samples
}

\author{
Abdenbi Mohand-Ousaid ${ }^{(0)}$, Sinan Haliyo ${ }^{\circledR}$, Stéphane Régnier, and Vincent Hayward (i)
}

\begin{abstract}
Micro-teleoperated interaction with biological cells is of special interest. The low fidelity of previous systems aimed at such small scale tasks prompted the design of a novel manual bilateral cell injection system. This systems employed the coupling of a null-displacement active force sensor with a haptic device having negligible effective inertia. This combination yielded a bilateral interaction system that was unconditionally stable even when the scaling gains were high. To demonstrate the capability of this system, two experiments were performed. A hard trout egg was delicately punctured and a small dye amount was injected in an embryo within a zebra fish egg without causing other forms of damage. The results demonstrate that the system let an operator dextrously interact with reduced reliance on visual feedback.
\end{abstract}

Index Terms-Biological cell manipulation, telerobotics and Teleoperation, human performance augmentation, haptics.

\section{INTRODUCTION}

$\mathbf{I}$ T IS well accepted that haptic feedback is highly valuable for the remote handling and manipulation of hostile, distant, small, virtual, or other inaccessible environments. Its importance is well established, see [1] or [2] for historical landmarks. When sufficient fidelity is available, force feedback can "transports" the sensorimotor-haptic-skills of human operator into the environments just mentioned, including manipulation at microscopic scales [3].

Specific interest in microscopic manipulation has recently grown greatly owing to its many applications in biology and in biomedical engineering, such as membrane mechanical testing in tissue engineering [4], [5] or manual injection of material into biological cells because of its relevance for in vitro fertilisation, intracytoplasmic sperm injection (ICSI), or pro-nuclei deoxyribonucleic-acid (DNA) injection.

Here, the specific focus is to describe a proof-of-concept application of manual injection of material into a cell using a stable

This work was supported in part by the French government research program "Investissements d'avenir" through the Robotex Equipment of Excellence ANR-10-EQPX-44 and in part by the Bourgogne Franche-Comté region project COMPACT. (Corresponding author: Abdenbi Mohand Ousaid.)

A. Mohand-Ousaid is with the Université Bourgogne Franche-Comté, FEMTO-ST Institute AS2M department, 25000 Besançon, France (e-mail: abdenbi.mohand@femto-st.fr).

S. Haliyo, S. Régnier, and V. Hayward are with Sorbonne Universités, Institut des Systèmes Intelligents et de Robotique, 75005 Paris, France (e-mail: sinan.haliyo@ sorbonne-universite.fr; stephane.regnier@ sorbonneuniversite.fr; vincent.hayward@ sorbonne-universite.fr).
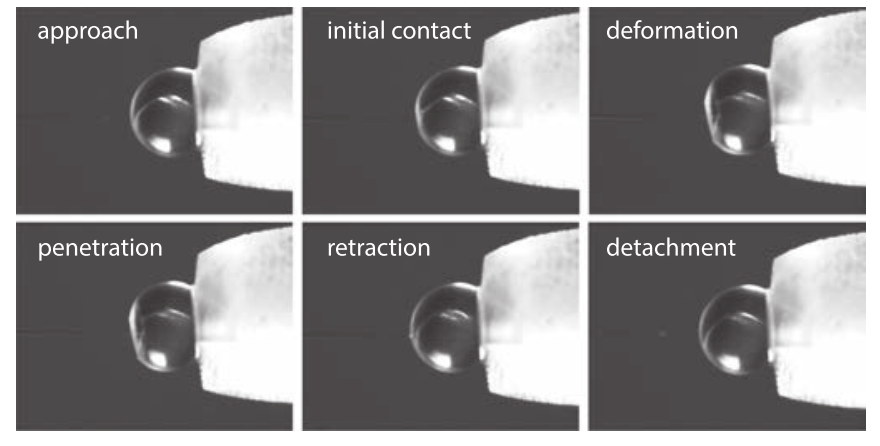

Fig. 1. Interaction phases. Cell injection comprises distinct phases: approach, initial contact, deformation, penetration, retraction, and detachment.

and highly transparent force feedback system capable of large force amplification gains without compromising stability. This system is applied to the experimental validation to puncturing an egg cell under bilateral manual control to deliver material inside the membrane.

At this scale, the physics of soft matter penetration by a sharp tool, with some differences, resemble penetration into macroscopic tissues [6], [7]. One important difference is that friction seems to play a negligible role. Other obvious differences are the time and length scale at which tissue damage (and repair) take place. Delivering material inside a cell may be decomposed into phases that correspond to specific modes of mechanical interactions: approach, initial contact, deformation, penetration, retraction, and detachment, all of which correspond to a distinctly different haptic sensations, see Fig. 1. Of particular perceptual interest are the quasi-instantaneous mechanical events, 'puncture' and 'detachment,' that signal phase transition from deformation to penetration and from retraction to move-away, that can be felt but cannot be seen.

Several systems integrating haptic feedback for bilateral interaction with the micro-world were described in the literature [8][12]. The quality of the feedback, however, is contingent to (i) the technique used to sense the forces of interaction (Van der Waals, Coulomb, capillary, deformation) taking place between the effector and the membranes, (ii) the haptic device employed to render this information and (iii) the bilateral coupling control algorithm. Each of these components multiplicatively (from cascading), and additively (from physical and numerical noise injection) degrades the quality of the interaction experienced by the operator.

System performance can be assessed through the notion of transparency which quantifies the ability of a bilateral robotic 
system to provide operators with accurate feedback free of artefacts [13]. When transparency is sufficiently high, a user has the sensation of interacting directly with a sample. Transparency provides users with useful assistance since filtered out, distorted, and noisy feedback impedes human performance rather than improving it. To quantify this observation, recall that the human sensorimotor space encompasses more than four orders of magnitude in displacement [14], effort [15], spans a range of frequencies of one kiloHertz [16], and can separate temporal events within $10 \mathrm{~ms}$ [17].

We built upon previous work on micro teleoperation [13], [18], to develop a high performance manual cell injection system. The system is based on high-fidelity bilateral coupling between a dual-stage haptic device and null-displacement active force sensor. This system is, to our knowledge, to-date the most efficient device to interact at small scales. On the user side, the haptic interface achieves no less than two orders of magnitude of improvement in transparency over existing designs, in terms of the magnitude of parasitic forces that reduce transparency, as well as in the ratio between the smallest perceptible torque and the largest one which can be generated. On the sample side, unlike the conventional passive sensors, the active sensor guarantees the complete capture of interaction forces. The combination of these two techniques brings several contributions to the problem of injection systems design.

a) The force measurement captures, in real time, the entire interaction forces between the micropipette and the sample, even when the interaction restitutes the stored energy;

b) The user can exercise expertise, judgment, and intuition since the highly transparent haptic device and the active force sensor are directly coupled without any intervening filter;

c) The proof-of-feasibility of a cell injection system with very high fidelity haptic feedback is presented.

We exemplify here the system performance through the injection of trout and zebrafish eggs of diameter $2000 \mu \mathrm{m}$ and $500 \mu \mathrm{m}$ respectively. The scaling gains were set to 3400 for force and 0.014 for displacement, amplifying the work exchanged between the probe and the sample by a factor of 2500 000. The design of our system could accommodate, without modification and without risks of instability, an exchanged work gain that could be a hundred-fold larger.

\section{RELATED WORKS}

Figure 3 shows the force sensor that we employed. It overcame the shortcomings of earlier sensors using an active force balance or compensation principle such that the probe was always kept to a null position when interacting with a load.

Various cell injection systems have been reported in the literature, with real or simulated samples. A generic structure, shown in Fig. 2, forms the basis of a wide range of injection systems with or without force feedback. As an early example, Sun et al., [19], proposed a micro-robotic cell manipulation system employing a multi-axis cellular force sensor to quantitate the mechanical property differences of the mouse Zona Pellucida (ZP) before and after fertilisation. Huang et al., [20],

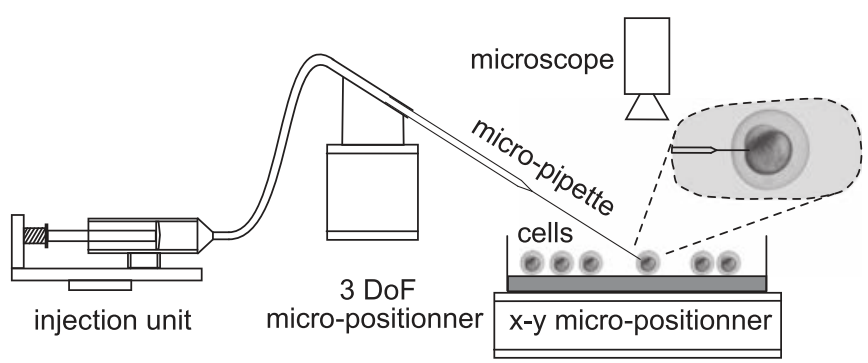

Fig. 2. Conventional cell injection system. This scheme illustrates the simplest and usual structure of cell injection system. Aided by visual feedback and micro-stages, the operator adjust manually the position of the micro-pipette according to the cell in order to achieve injection task.

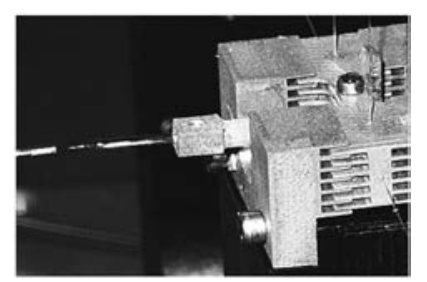

Fig. 3. Active Force Sensor. A frictionless mobile probe was feedbackcontrolled to keep a fixed position through a comb-drive actuator. The force generated by the controller to compensate external efforts gave the measurement.

developed a cell injection system combining visual processing and force measurement for automatic batch injection of suspended cells. Reference [21] describes a micro-manipulation system for full automatic batch micro-injection of zebrafish embryos. Liu et al., [22] also developed an automated robotic injection system for delivering cell impermeable compounds into mouse zygotes. These systems, aimed at batch processing, lack haptic feedback and thus do not take advantage of the sensorimotor abilities of human operators.

Cho and Shim, [23], developed a system to measure force in bio cell injection and transmit it to operator through haptic feedback. Pillarisetti et al. [8], described a device measuring forces in the $\mu \mathrm{N}$ range providing force feedback in real time. This system used a PHANToM interface as a master, PVDF film as a force sensor, and had a micropipette for injection. Recently, Guangwei [9], developed a novel piezo-driven cell injection system with force feedback. These systems increased the rate of success of the injection process. However, force measurements and force feedback were typically noisy and tainted by artefacts. Owing to the high homothetic gains required by interacting with the micro-world system transparency is difficult to assess. In addition, most of commercial haptic interfaces employed are affected by inertia and friction. Force sensing typically based on the passive measurement of the deformation of an elastic element enforces tradeoffs between stiffness, sensitivity and structural response characteristics.

\section{High-Transparency Cell InJeCtion System}

The transparency of bilateral systems depends (1) on the measurement of the interaction force translated into a signal; (2) the bilateral control scheme where this signal is scaled up or scaled down; and (3) on the haptic interface where the signal is 


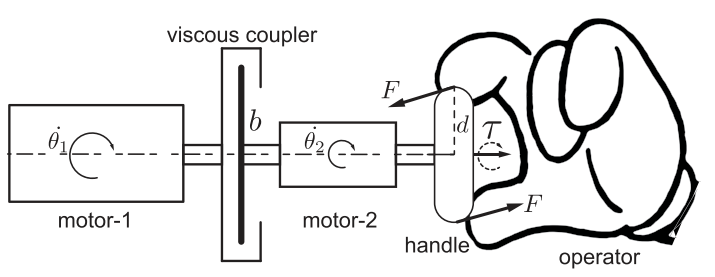

Fig. 4. Dual-stage haptic feedback device. The output torque is provided by the velocity of the large motor, through a viscous coupler, and the small one compensates the following error.

translated back into a force. In an ideal case, the force felt by the user would be pure homothetic amplification of the interaction force, entirely devoid of artefacts introduced by the mechanics of the sensor, by the haptic device, and by defects introduced by the numerical coupling.

The cell injection system described here approached these specifications. Because the apparent stiffness of the probe was set by active feedback to very high values, the work exchanged with the environment was reduced a negligible amount. Within the sensor bandwidth, interaction forces could be tracked in their entirety. This feature also eliminated undesirable behaviours when dealing with non-contact forces, which is a common occurrence in the micro-world. Coupled to this sensor is a haptic interface which, owing to its dual-stage design, approximated closely a pure force generator, with very low effective inertia, thus reducing stored kinetic energy. The coupling of these two elements yielded a system that was unconditionally stable under a wide range of operating conditions even when the scaling gains were high.

\section{A. Force Measurement}

The sensor we employed overcame the shortcomings of earlier sensors using an active force balance or compensation principle such that the probe was always kept to a null position when interacting with a load. As a result, the internal mechanics of the probe did not intervene in the measurement and it was possible to track complete force/distance curves at all points in space, avoiding 'jump-to-contact' behaviours. A similar sensor was employed successfully to measure and track small scales interactions like pull-in and pull-off forces during the interaction of the leg of an ant with a droplet of water [18].

Because this sensor operated with a highly sensitive electrostatic comb-drive actuator, it was placed in an enclosure to isolate it from acoustic perturbations. The sensor was able to measure forces within a range of $\pm 400 \mu \mathrm{N}$ with a resolution of $\sim 0.4 \mu \mathrm{N}$ and a bandwidth of $10 \mathrm{~Hz}$.

An additional feature of an active feedback force sensor is its ability to measure forces around a fixed offset, thus increasing its dynamic range. This range was amply sufficient to cover the forces in play during microinjections. Readers can find more details about this sensor in [24].

\section{B. Haptic Feedback}

We used a haptic device interface with 1-DOF as depicted in Fig. 4. The interface was connected to the force sensor through

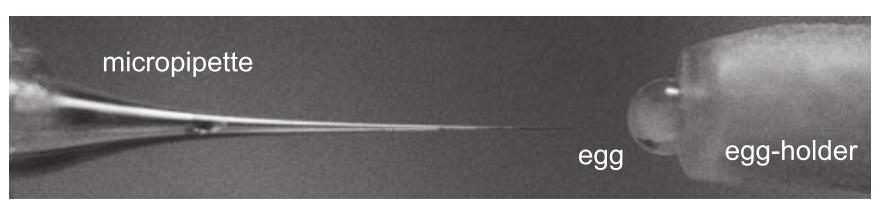

Fig. 5. Micropipette and egg holder. The pipette had a diameter of $\sim 10 \mu \mathrm{m}$ and egg specimens were immobilised by suction.

direct haptic coupling. Unlike conventional haptic interfaces using a single stage electromagnetic motor, this interface was based on dual-stage architecture where a large motor and a small motor are coupled via a viscous coupler operating on the basis of eddy currents. This dual arrangement made the primary output torque a highly accurate reflection of the slip velocity in the coupler. Velocity feedback was utilised to eliminate the effect of the large inertia. The output torque is mainly provided through the velocity of the large motor and the small one compensates the following error. The device hence combines the high-frequency response of a small motor with a large torque, resolving the tradeoff between a torque magnitude and better dynamic tracking performance. The interface achieved low inertia and a wide dynamics range covering four orders of force magnitude $(5.2 \mathrm{mN}$ to $5.7 \mathrm{~N}$ ). It is worth to note that the ability to display forces at such lower boundary allows the user to detect smallest details such as a particular texture or slight variations of vibration patterns, which often carries quite useful information. A practical example is the differences felt when penetrating the chorion vs the embryo in the zebrafish egg injection task. Moreover, the interface frequency response was flat in a range of $1 \mathrm{kHz}$. Readers can refer to [18], [25], [26] for more details about earlier versions of this device.

Briefly, referring to Fig. 4, the output torque, $\tau$, delivered by the device to the hand of the operator was the sum of the torque transmitted by the coupler, which was proportional to the difference of velocities between the two motors, and the torque of the small motor, $\tau_{2}, \tau \approx b\left(\dot{\theta_{1}}-\dot{\theta_{2}}\right)+\tau_{2}$, where $b$ is the viscous coefficient of the coupler and where $\dot{\theta}_{1}, \dot{\theta_{2}}$ are the velocities of the large and the small motors, respectively.

Such configuration simultaneous achieve very low effective inertia as seen from the hand of the operator, a wide dynamic range owed to the large motor, and wide bandwidth afforded by the small motor. Friction can also be kept low. The interface parameters were carefully calibrated.

This device is particularly adapted to dynamics of the microscale, where small forces at high frequencies and larger ones coexist: for example the force while puncturing the membrane, and the friction on the probe during its traversal. These components are equally important to transmit to the user. This is quite impossible to achieve on an off-the-shelf device, which would either saturate large forces to reflect correctly small ones, or drown small forces in the noise to properly reflect large ones.

\section{Micropipette Holder}

The injection micropipette holder was supported by two conventional motorised micro-positioning stages. The first had axes 


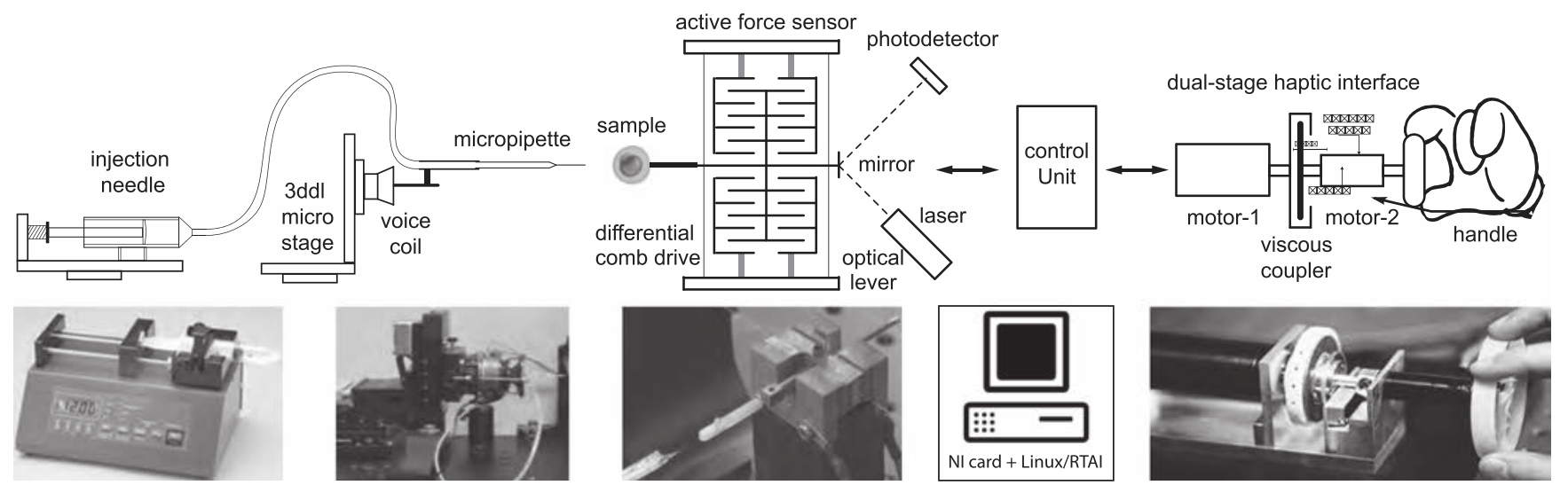

Fig. 6. Complete injection chain comprising a pressure generator, a micropipette holder, an active micro-force sensor, a control unit, and dual-stage haptic device.

and provided a range of $25 \mathrm{~mm}$ with $0.04 \mu \mathrm{m}$ resolution (Sutter MP-285). The second, for the fine positioning, was simply an electromagnetic voice coil transducer taken from a loudspeaker.

\section{Injection Unit}

The injection unit comprised a tiny micropipette for injection and micro pump (AL-1000, World Precision Instruments) to control the volume of injected dye. The micropipettes were obtained by hot-pulling hollow glass cylinders of diameter $1.5 \mathrm{~mm}$ (P-1000 Micropipette Puller from Sutter). Fig. 5 illustrates the micropipette which had a diameter of $\sim 10 \mu \mathrm{m}$ and contained coloured dye. The probe of the sensor carries a specimen holder, which immobilised the egg by suction. A digital microscope camera enabled the operator to view the sample-micropipette interaction in real time and a camera with appropriate optics is used for recording videos and images.

\section{E. Control Unit}

The system was controlled through a PC running real-time operating system Linux/RTAI. The system is interfaced to the computer via a NI DAQ PCI 6259 card (National Instruments). Two discrete-time controllers were implemented and ran in a single loop at a rate of $1 \mathrm{kHz}$. A polynomial controller and a robust $\mathrm{H}_{\infty}$ controller were applied to the haptic device and the micro-force sensor, respectively. The position of the handle was scaled down and was used as a position set point to the transducer carrying the micropipette holder. The force measured by the sensor was scaled up and used as force set-point by the haptic interface control system.

\section{F. System Stability and Transparency}

While the whole system was adapted from the micro teleoperation system described in [13], [18], some key modifications were made to achieve cell injection tasks. Despite these modifications, the stability and transparency analyses were similar. It included an impedance criterion for transparency analysis and the application of the Llewellyn passivity criterion or stability analysis [13], [27]. Thus in this new configuration, quasi-perfect transparency was achieved. The impedances were equal when the scaling gains were applied. Fig. 6 shows the integrated system which was placed in a controlled environment to ensure the most favorable conditions.

\section{EXPERIMENTS}

We evaluated the ability of our set-up to facilitate microrobotic biological applications such as cell injection. For this, we tested the ability of the system to scale the physics of micro interactions such that the different interaction phases: contact, puncture, and so on, could be directly experienced during an egg injection task. Two experiments have been carried out on a trout egg and zebrafish egg, respectively. The first sample, is often considered to be hard. It has one layer and its content is dense. The second sample is considered to be soft, has two layers and its content is gelatinous.

Fig. 7 provides a representation of the visual feedback available to an operator. The different phases might be easily identified visually but the onset of transitions, which are crucial to be detected for a successful completion of the task, are invisible. They must inferred, hence with considerable delay, from the identification of the long lasting phases.

\section{A. Trout Egg}

A complete interaction sequences in reported in Fig. 8. The long lasting phases are clearly visible. Upon contact, the external layer of the egg deformed until the probe penetrated the external layer. The probe was then retracted to release it. The time scales were such that the long lasting phases as well as the transitory phases could be clearly felt. After the contact the interaction force increases and then drops down suddenly when the external layer was punctured. The error between the estimation of the transmitted force and the amplification of the measured force was smaller than $1 \%$ over the whole sequence experiment demonstrating the high degree of transparency of the system.

\section{B. Zebra Fish Egg}

The experiment included the injection of dye inside a zebrafish egg specimen, assisted by force feedback. Such specimen is a common animal model used in biology research because it 


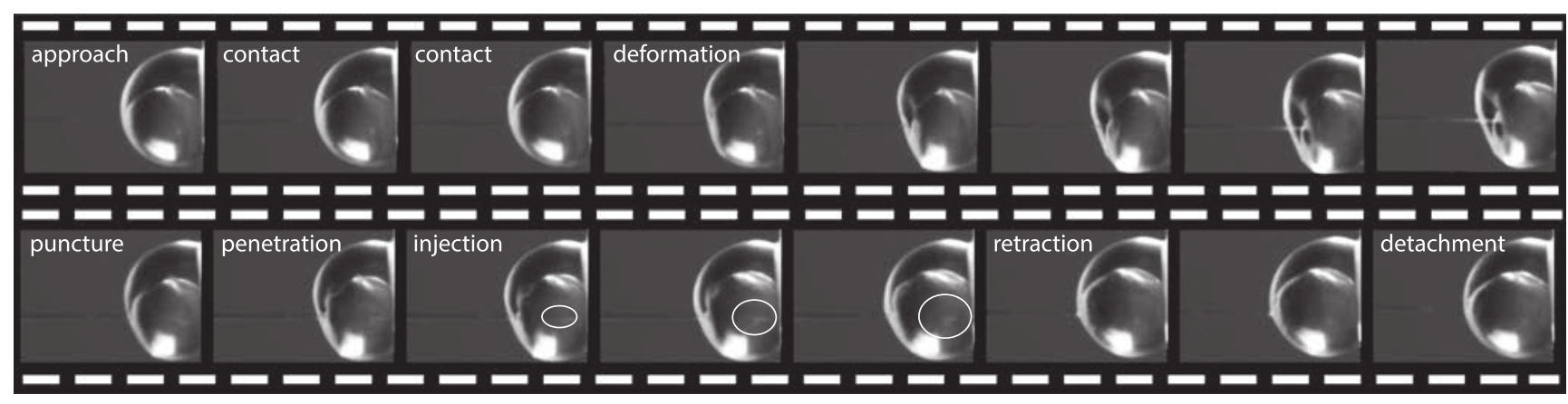

Fig. 7. Visual feedback during the injection sequence in a zebra fish embryo. Two membranes had to be transpierced: the outer Chorion, and the inner Vitelline membrane. Those two structures exhibited very different stiffness and mechanical rupture properties.
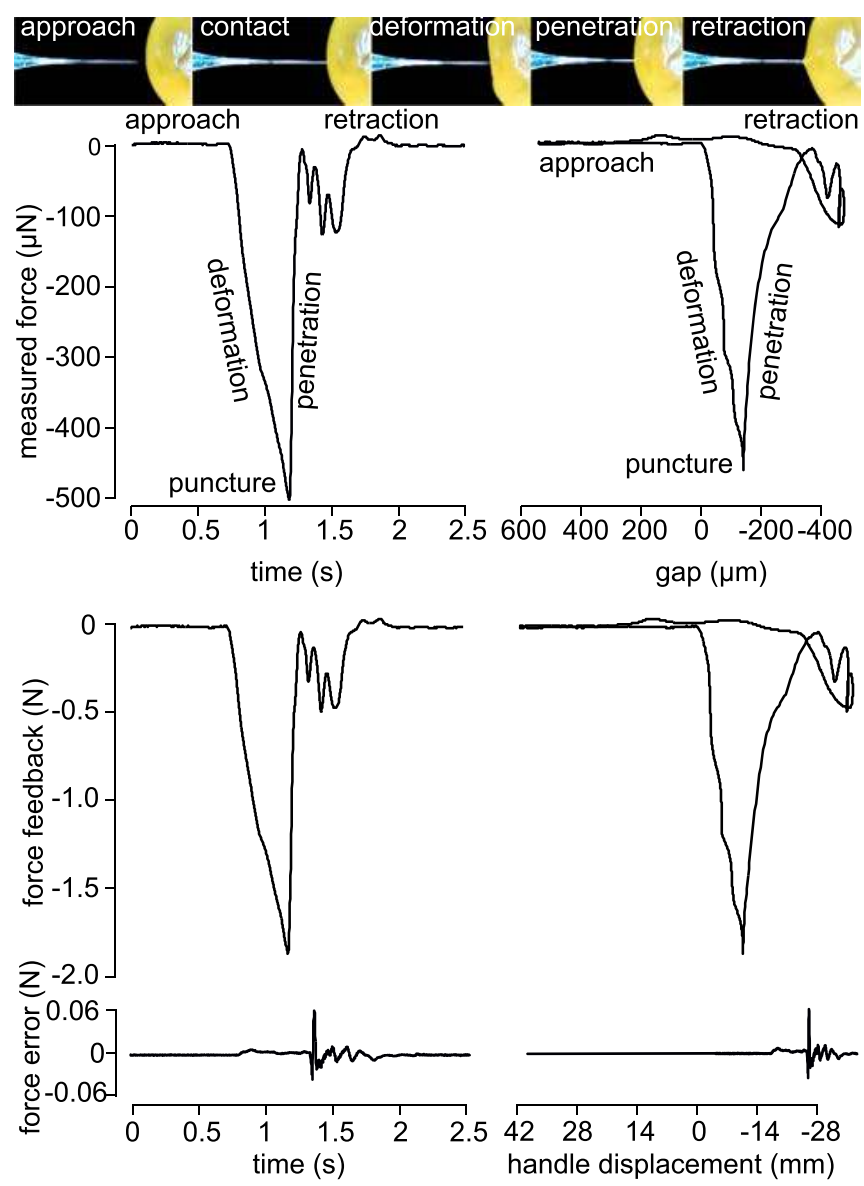

Fig. 8. Interaction with a hard trout egg. The force reflected to the user (central curve) was near-perfect linear amplification of the measured interaction (top curve).

has about $96 \%$ of the same DNA as others animals. It is also used because the egg is optically translucide which allows for direct visual observation of the injection process among other experimental options. Zebrafish eggs are easily available, develop quickly, and allow for external fertilisation [20]. Their diameter is approximately $2 \mathrm{~mm}$ or less.

The objective of the test was to inject the dye in an embryo and to verify that it remained there. An egg containing an embryo was sucked in the holder that was supported by the probe of the sensor. The task was to manually guide the micropipette to
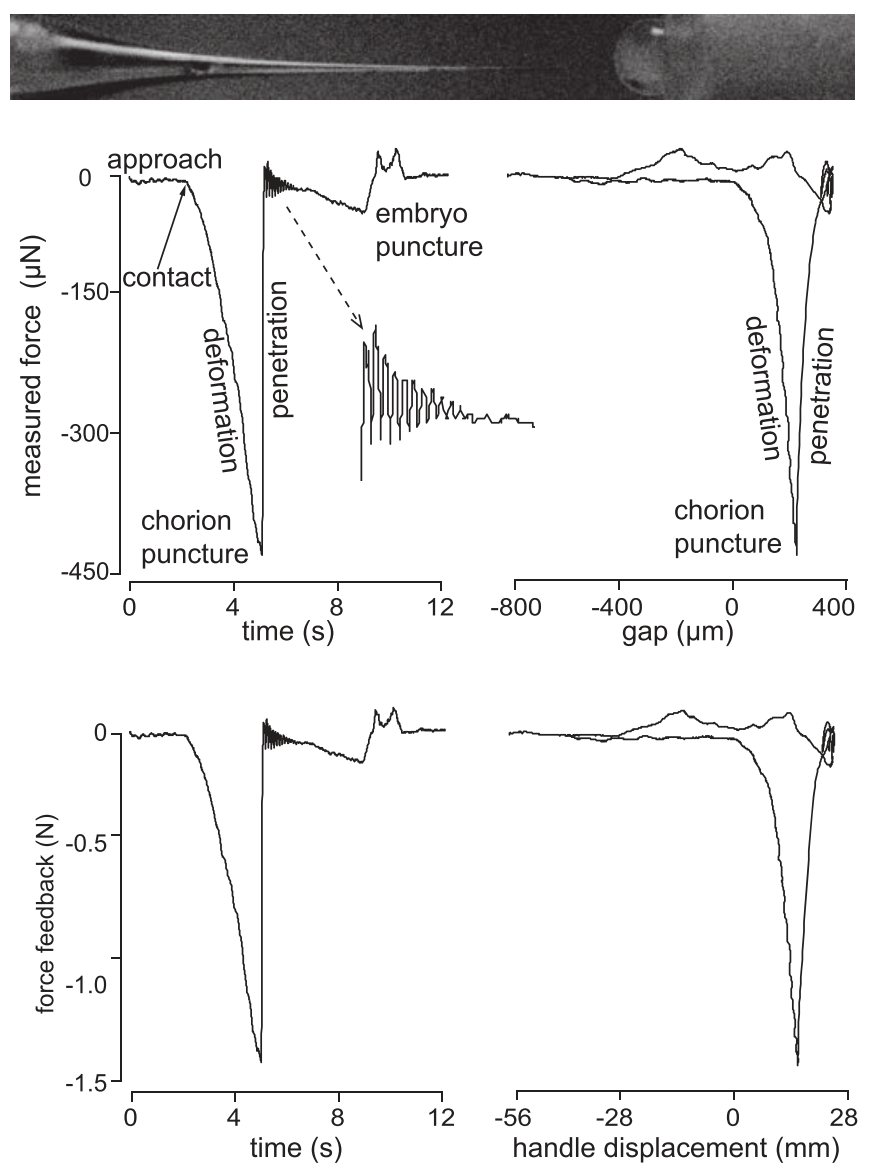

Fig. 9. Injection of dye in a zebra fish embryo. As on the trout egg experiment, the systems exhibited a very high transparency between micro and macro-scales.

penetrate the chorion and then the embryo, and the fulfilment of the objective could be assessed by observing visually that the dye was injected directly in the embryo.

Upon contact, the chorion resisted penetration much less than when pushing against a trout egg as can be observed in Fig. 9. After penetration, the dye was injected inside the embryo and the micropipette was retracted. The small, rapid oscillations that can be observed just after puncture are due to the limited sensor bandwidth combined with the fact that the substance inside the egg was less dense compared to the trout egg which provided more damping. The interaction force rose again when the pipette 
came into contact with the embryo, yet to a smaller extent than when pushing against the chorion. Yet it was detectable by feel and the transitory sub-punctures indicated that the dye injection could take place. A skilled operator could then anticipate the occurence of embryo penetration from the clear haptic signal provided by the successive punctures. Referring back to Fig. 7, the operator could proceed with injection with the certainty that the dye (indicated by a white oval) when diffusing in the embryo remained there.

\section{CONCLUSION}

The system described was optimised for egg injection tasks. It could transmits and amplify faithfully the measured interaction forces to the operator, despite a mechanical work amplification by a factor of 2500 000, and provide assistance during an injection task that was not available visually.

The cell injection system described herein enabled direct access to the micro-scale interactions. Actual practice demonstrated that the system made it possible for an operator to inject these samples dextrously and without excessive reliance on visual feedback. The clearly sensed microscopic forces enabled the operator to spontaneously differentiate the phases of the interaction, assisting in rapid execution of the injection process.

This unprecedented advance was achieved by a combination of quasi-perfect haptic transparency and unconditional stability of the bilateral micro-teleoperated system. This approach provided spontaneous control over the process and enabled the operator to minimise the damage made to the tissues. The approach can be easily extended to other more complex tasks such as the injection of very small samples in specific target areas, or to the selective extraction of genetic material.

\section{ACKNOWLEDGMENT}

Authors wish to thank Dr. Francois Giudicelli (CNRS researcher) for providing zebrafish eggs and Dr. Nicolas David (CNRS researcher) for advising on dye injection.

\section{REFERENCES}

[1] R. C. Goertz, "Fundamentals of general purpose remote manipulators," Nucleonics, vol. 10, no. 11, pp. 36-42, 1952.

[2] A. K. Bejczy, "Sensors, controls, and man-machine interface for advanced teleoperation," Science, vol. 208, no. 4450, pp. 1327-1335, 1980.

[3] R. Hollis, S. Salcudean, and D. Abraham, "Toward a tele-nanorobotic manipulation system with atomic scale force feedback and motion resolution," in Proc. IEEE Micro Electro Mech. Syst., Proc., An Investigation Micro Struct., Sensors, Actuators, Mach. Robots, Feb. 1990, pp. 115-119.

[4] K. Kurpinski, J. Chu, C. Hashi, and S. Li, "Anisotropic mechanosensing by mesenchymal stem cells," Proc. Nat. Acad. Sci., vol. 103, no. 44, pp. 16 095-16 100, 2006.

[5] S. Suresh, "Biomechanics and biophysics of cancer cells," Acta Materialia, vol. 55, no. 12, pp. 3989-4014, 2007

[6] M. Mahvash and V. Hayward, "Haptic rendering of cutting: A fracture mechanics approach," Haptics-e, vol. 2, no. 3, pp. 1-12, 2001.
[7] S. Greenish, V. Hayward, V. Chial, A. Okamura, and T. Steffen, "Measurement, analysis and display of haptic signals during surgical cutting," Presence: Teleoperators Virtual Environ., vol. 6, no. 11, pp. 626-651, 2002.

[8] A. Pillarisetti, M. Pekarev, A. Brooks, and J. Desai, "Evaluating the effect of force feedback in cell injection," Autom. Sci. Eng., IEEE Trans. on, vol. 4, no. 3, pp. 322-331, Jul. 2007.

[9] G. Wang and Q. Xu, "Design and development of a piezo-driven microinjection system with force feedback," Adv. Robot., vol. 31, no. 10, pp. 1-11, 2017.

[10] A. Ghanbari, B. Horan, S. Nahavandi, X. Chen, and W. Wang, "Haptic microrobotic cell injection system," Syst. J., IEEE, vol. 8, no. 2, pp. 371-383, Jun. 2014.

[11] Z. Nan, Q. Xu, Y.Zhang, and W. Ge, "Force-sensing robotic microinjection system for automated multi-cell injection with consistent quality," IEEE Access, vol. 7, pp. 55 543-55 553, 2019.

[12] Y. Wei and Q. Xu, "Design and testing of a new force-sensing cell microinjector based on soft flexure mechanism," IEEE Sensors J., vol. 19 no. 15, pp. 6012-6019, Aug. 2019.

[13] A. Mohand-Ousaid, S. Haliyo, S. Régnier, and V. Hayward, "A stable and transparent microscale force feedback teleoperation system," IEEE/ASME Trans. Mechatronics, vol. 20, no. 5, pp. 2593-2603, 2015.

[14] S. P. N. Singhy and C. N. Riviere, "Physiological tremor amplitude during retinal microsurgery," in Proc. IEEE 28th Annu. Northeast Bioengineering Conf., 2002, pp. 171-172.

[15] G. Baud-Bovy and E. Gatti, "Hand-held object force direction identification thresholds at rest and during movement," in Haptics: Generating and Perceiving Tangible Sensations, ser. Lecture Notes in Computer Science, A. Kappers, J. van Erp, W. B. Tiest, and F. van der Helm, Eds, Berlin, Germany: Springer, 2010, vol. 6192, pp. 231-236.

[16] R. T. Verrillo, "Psychophysics of vibrotactile stimulation," J. Acoustical Soc. Amer, vol. 77, no. 1, pp. 225-232, 1985.

[17] G. A. Gescheider, "Resolving of successive clicks by the ears and skin," J. Exp. Psychol., vol. 71, no. 3, pp. 378-381, 1966.

[18] A. Mohand-Ousaid, G. Millet, S. Régnier, S. Haliyo, and V. Hayward, "Feeling what an insect feels," PLOS ONE, vol. 9, no. 10, 2014, Art. no. e108895.

[19] Y. Sun, K.-T. Wan, K. P. Roberts, J. C. Bischof, and B. J. Nelson, "Mechanical property characterization of mouse zona pellucida," Nanobiosci., IEEE Trans., vol. 2, no. 4, pp. 279-286, 2003.

[20] H. Huang, D. Sun, J. Mills, and S. Cheng, "Automatic suspended cell injection under vision and force control biomanipulation," in Proc. Robot. Biomimetics, ROBIO. IEEE Int. Conf., Dec. 2007, pp. 71-76.

[21] Z. Lu, P. C. Chen, J. Nam, R. Ge, and W. Lin, "A micromanipulation system with dynamic force-feedback for automatic batch microinjection," J. Micromechanics Microengineering, vol. 17, no. 2, pp. 314, 2007.

[22] X. Liu et al., "Automated microinjection of recombinant bcl-x into mouse zygotes enhances embryo development," PloS one, vol. 6, no. 7, 2011, Art. no. e21687.

[23] S.-Y. Cho and J.-H. Shim, "A new micro biological cell injection system," in Proc. Intell. Robots Syst., Proc. IEEE/RSJ Int. Conf., vol. 2, Sep. 2004, pp. $1642-1647$.

[24] A. Mohand-Ousaid, S. Haliyo, S. Régnier, and V. Hayward, "Micro-force sensor by active control of a comb-drive," in Int. Conf. Adv. Intell. Mechatronics, 2013, pp. 612-617.

[25] G. Millet, S. Haliyo, S. Régnier, and V. Hayward, "The ultimate haptic device: First step," in Proc. Third Joint Eurohaptics Conf. Symp. Haptic Interfaces Virtual Environ. Teleoperator Syst.. World Haptics, 2009, pp. $273-278$

[26] A. Mohand-Ousaid, G. Millet, S. Haliyo, S. Régnier, and V. Hayward "Haptic interface transparency achieved through viscous coupling," Int. J. Robot. Res., vol. 31, no. 3, pp. 319-329, 2011.

[27] A. Mohand-Ousaid, A. Bolopion, S. Haliyo, S. Régnier, and V. Hayward, "Stability and transparency analysis of a teleoperation chain for microscale interaction," in Proc. IEEE/Int. Conf. Robot. Autom., 2014, pp. 5946-5951. 